\title{
Digital Smile Design in interdisciplinary and orthodontic dental treatment planning
}

\author{
G. Finelle \\ 1 Attached to Charles Foix Hospital, Ivry-sur-Seine - Esthetic Consultation (Bioteam Paris) \\ 2 Exclusive private practice in implantology and prosthesis
}

\begin{abstract}
It is a fact that esthetic rehabilitation is in high demand. To meet patient expectations, the dental community tends to use digital processes and modern communication techniques to reinforce treatment predictability and overcome esthetic challenges. Currently, digital smile design (DSD) processes offer the whole dental team including the orthodontist a powerful digital approach to improve esthetic analysis, team communication, and collaborative treatment planning, guided by facial harmony.
\end{abstract}

\section{KEYWORDS}

Digital Smile Design, DSD, mock-up, virtual 3D diagnosis, facial analysis, interdisciplinary, communication, esthetic dentistry, digital orthodontics

\section{INTRODUCTION}

In their seminal article in 2002, Magne and Belser ${ }^{1}$ described, through a physiological puzzle, the existing and vital relationship between function, biology, mechanics, and esthetics. If the esthetic parameter is not the one that will ensure the longevity of treatment, it remains the only criterion on which our patient can judge the quality of the work performed, making it an integral part of "therapeutic success."
The esthetic challenges represented by previous multidisciplinary rehabilitations would not have been successful without perfect communication between the various members of the therapeutic team (prosthetic practitioner, implantologist, orthodontist, laboratory prosthetist). ${ }^{2}$ Difficulties often appear at a late stage in the project often because of initially unpredictable obstacles, and most of the time lead

Address for correspondence:

Gary Finelle - 59, avenue de la Bourdonnais - 75007 Paris

E-mail: garyfinelle@gmail.com

Article received: 10/02/2017. Accepted for publication: 28/02/2017.

This is an Open Access article distributed under the terms of the Creative Commons Attribution License (http://creativecommons.org/licenses/by/4.0), which permits unrestricted use, distribution, and reproduction in any medium, provided the original work is properly cited. 
to end-of-treatment compromise situations which are more or less well accepted by the patients.

In many areas of activity, project validation is formalized in the form of a mock-up, sketch, or prototype, which is accessible to all professionals (regardless of specialty) involved in the project.

Although the digital environment is becoming increasingly essential in the dental industry and more specifically in esthetic dentistry and orthodontics, smile preview techniques ${ }^{3}$ such as the Digital Smile Design (DSD), developed by Dr. Christian Coachman ${ }^{4}$ or VEP $^{5}$ (Oral Laboratory Esthetics, Didier and Hélène Crescenzo) are essential reference tools (generally led by the practitioner in charge of esthetic rehabilitation) to understand, plan, communicate, and share therapeutic projects with the team and avoid pitfalls or possible unanticipated compromises.
In other words, the smile preview techniques respond to the need to bring together all the diagnostic elements collected by the team in a multidisciplinary project to make the treatment as predictable as possible.

This article, written for the orthodontic community, has the following aims:

- shed light on the concepts and uses of recent smile preview techniques in multidisciplinary management;

- describe the clinical and informal protocol through a simple and didactic orthodontic case;

- illustrate through concrete examples the principle of Test Drive (mock-up);

- give a glimpse of the developments and the perspectives of Smile Design in orthodontic care.

DSD is, to date, the most common tool. Thus, the details of this article will focus on this technique only.

\section{ESTHETIC ANALYSIS AND DIAGNOSTIC VISION}

During the initial clinical examination, many esthetic parameters must be considered to establish a suitable treatment plan. It is not simply a question of listing them, but of rigorously analyzing them to identify the difficulties and anticipate possible compromises.

DSD is based on esthetic analysis from two-dimensional images. Therefore, the first step of the protocol is to take a series of photographs (facial and intraoral) and videos during the consultation session.

The primary objective of DSD is to improve the understanding of the case and the diagnostic vision by tracing the reference lines of the face (pupillary line, horizontal line of reference, median line of the face, smile line...; Fig. 1). The superposition of the face with the intraoral view allows an analysis of the dental arches (interincisal axis, position of the collars, position of the incisal margins, position of the papillae...) within the orofacial sphere ${ }^{6}$ (Fig. 2).

The pathways and shapes are made using a keynote digital presentation software or PowerPoint (Fig. 3). The order of the slides required to design DSD always follows the same procedure described below. 


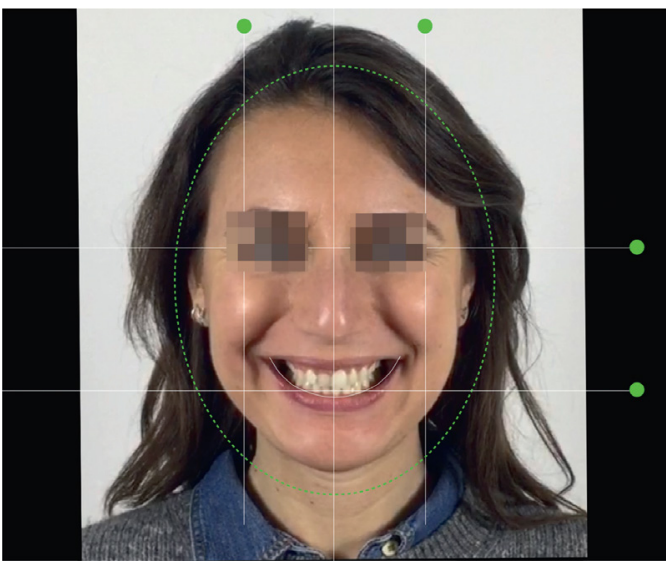

Figure 1

The image of the face with a forced smile, positioned against the background image, allows us to determine the ideal horizontal plane as well as the interincisal background.

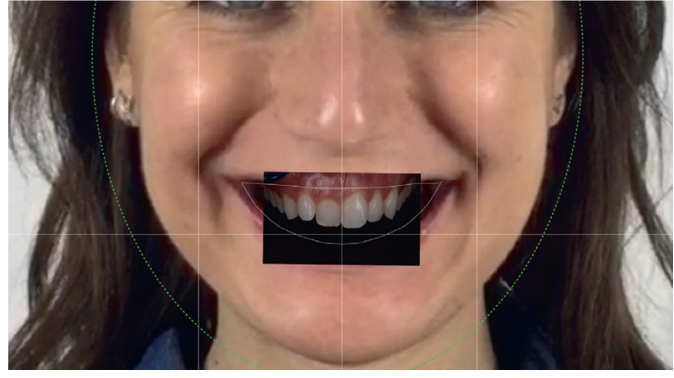

Figure 2

The intraoral photo is merged with the facial photo to transfer the information from the orofacial context to the intraoral snapshot.

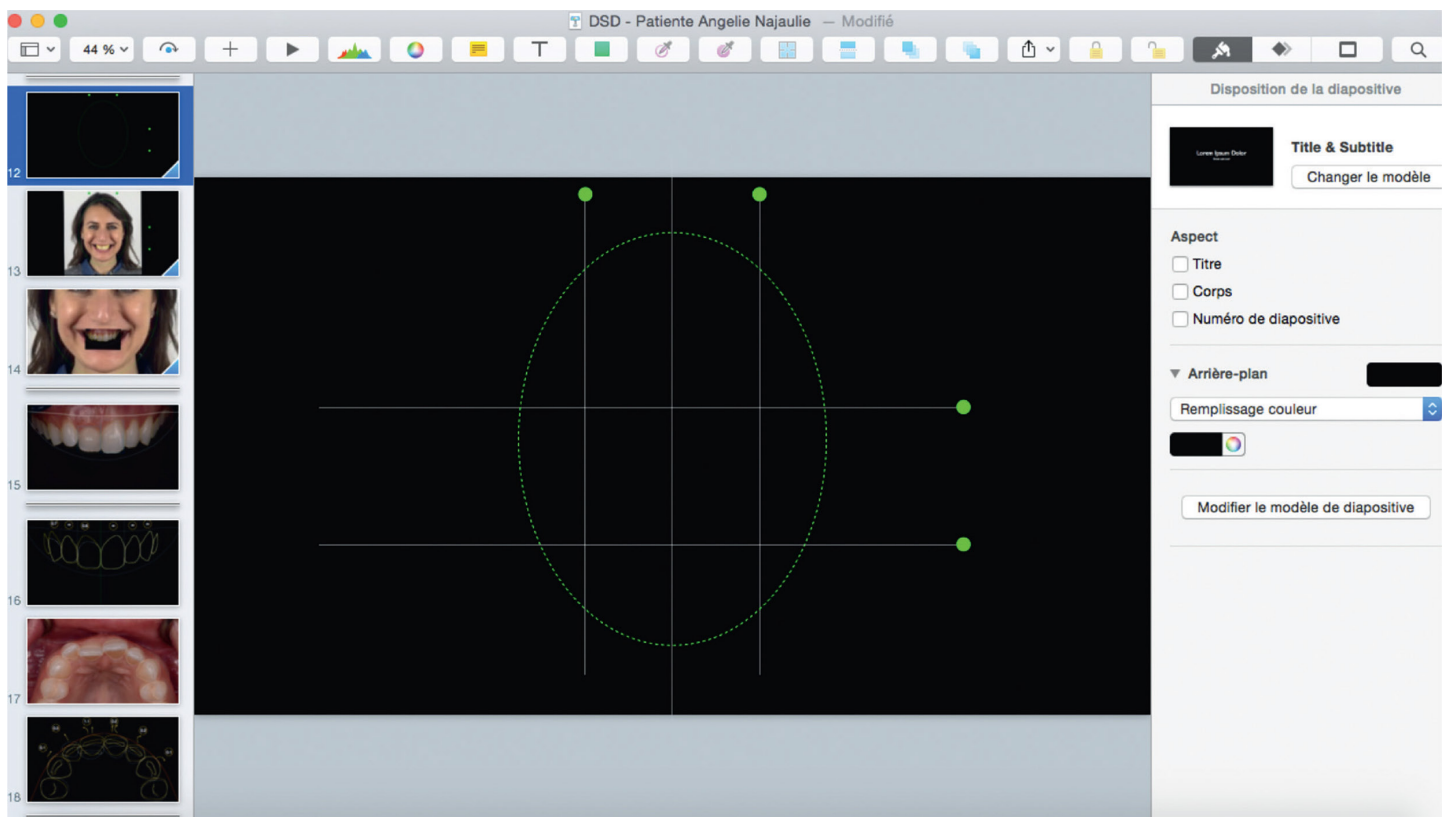

Figure 3

Keynote presentation software is used to organize DSD. The first slide contains the information on the bi-pupillary line (horizontal) and the median facial line (vertical). 


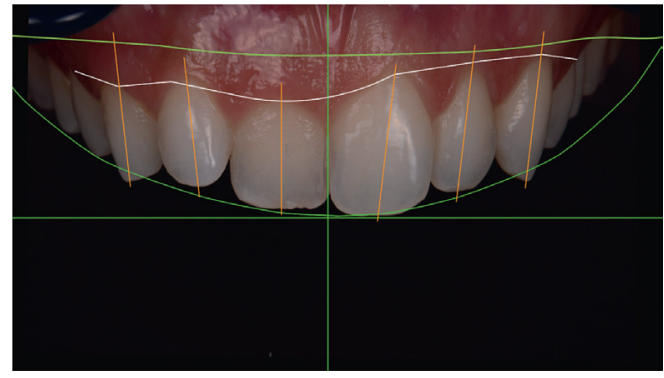

Figure 4

The facial information drawn in green (position of the smile line, smile curve, facial median line, horizontal plane) now appear on the anterior vestibular image. Dental information can also be added to help improve esthetic visualization and optimize communication. Here the dental axes (orange) and the positions of the collars (white) are highlighted.

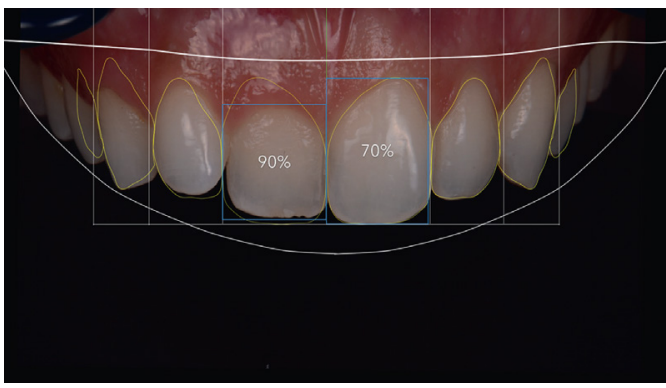

Figure 6

The smile design is achieved in accordance with the treatment objectives defined by the results of the analysis. Frontal View, teeth 11 and 13 must be lengthened.

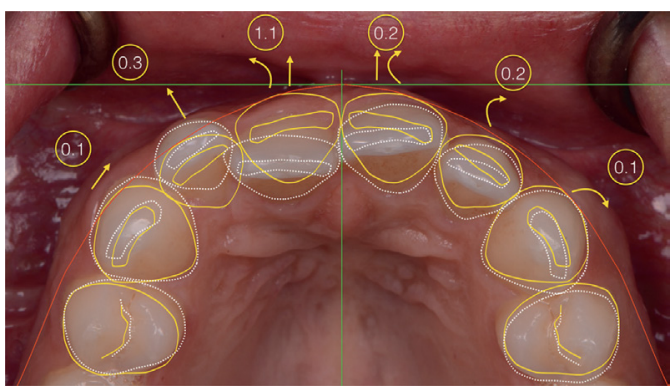

Figure 8

Occlusal view, when treatment involves prior orthodontic care, the ideal position of the teeth (yellow lines) is drawn, with the associated movements being annotated with arrows.

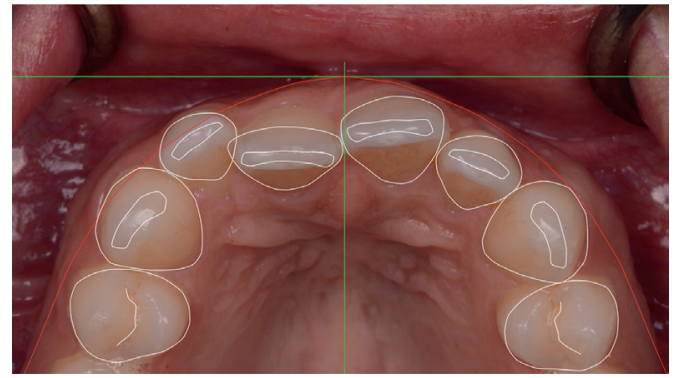

Figure 5

The occlusal view makes it possible to objectify the depositions relating to the anterior maxillary congestion.

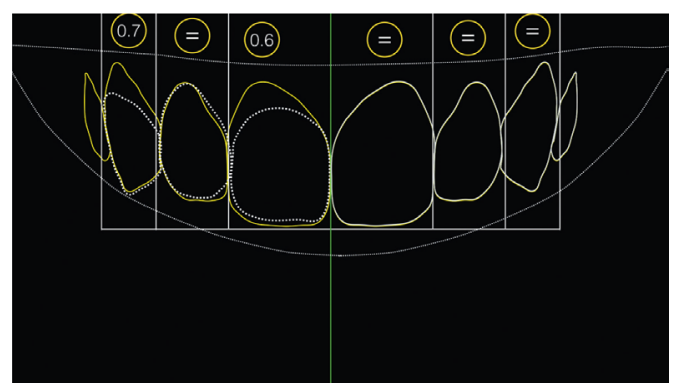

Figure 7

Precise quantitative information (measures in $\mathrm{mm}$ ) are sent to the laboratory prosthetist who will perform the set up. This diagram illustrates the project (yellow) in comparison with the current position.

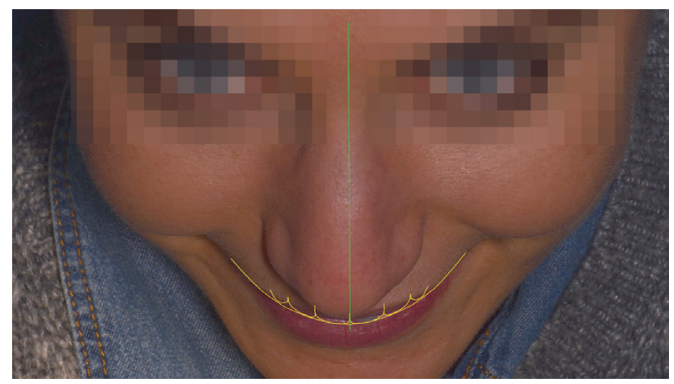

Figure 9

The "12 o'clock" view allows the vestibular projection of incisal margins to be judged in relation to the position of the lower lip. 


\section{PLANNING AND DESIGN OF THE DSD}

For didactic purposes, the case selected to describe DSD step by step is deliberately chosen because it is a simple case.

A simple case. The more complex the case seems, the more useful the use of smile preview techniques (Figs. 4-9) ${ }^{7}$

The detailed analysis file can be made available to the therapeutic team on a "cloud" system (Dropbox, Google Cloud...) allowing an effective and instantaneous collaborative approach between the different practitioners in charge of the treatment. The digital project can then be shared, completed, and carried out by the various specialists involved in the treatment.

In the majority of cases, it seems relevant that DSD be undertaken by the practitioner responsible for the overall management of the treatment and made available to the various people involved.
The purpose of the virtual analysis is formalized in the last "slide" of presentation and available in the form of a virtual synthesis or "smile frame" including the three key photos (vestibular view, occlusal view, 12 o'clock view) as well as the key information concerning the shape, position, and proportion of the teeth involved in the project (Figs. 10, 11). DSD is also complemented by several short video recordings for dynamic and functional analysis.

This summary scheme is the reference from which the whole team multidisciplinary team ${ }^{8}$ can

- indicate the management of the various stakeholders (prosthetist practitioner, implantologist, orthodontist, periodontist);

- guide therapeutic decisions;

- coordinate the treatment between the different departments;

- ensure the process is validated at the end of treatment.

\section{FROM THE VIRTUAL PROJECT TO CREATING THE MOCK-UP (Or TEST DRIVE)}

Traditionally, esthetic projects which involve prosthetics or orthodontics are made by dental prosthetists based on an often limited amount of oral or written information. Quite often the project (in the form of wax up or set up), once tried in the mouth in the form of a mock-up, does not fully achieve the treatment aims. The dilution of certain information from the orofacial context between the clinic and the laboratory is often the cause.

It is assumed that the esthetic rehabilitation must take into account the reference information constituting the harmony of the face (horizontal reference plane, facial median line, position and shape of teeth on the dental arch, shade.

The real difficulty lies in the faithful transfer of photographic data to the set up (or wax up) and therefore on the final result. Smile preview techniques have been developed to make these information transfers more reproducible.

Thus, the design of the set up or wax up (conventional or digital) is established from 2D photos and a digital virtual project. 


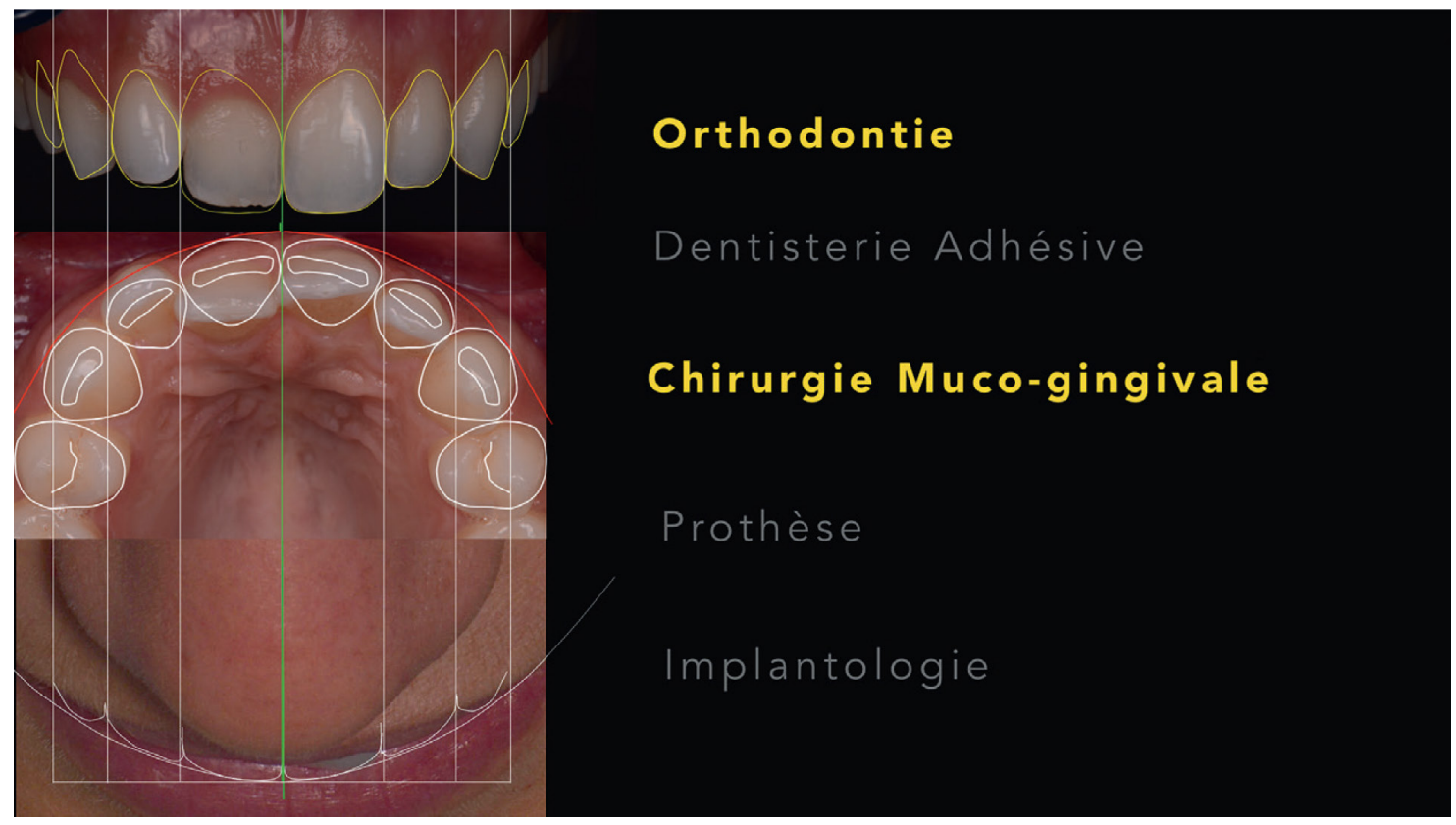

Figure 10

The analysis indicates initial orthodontic treatment. Then, after reassessment, a gingival site guided by the attached project will reposition the collars of 11 and 13 apically.

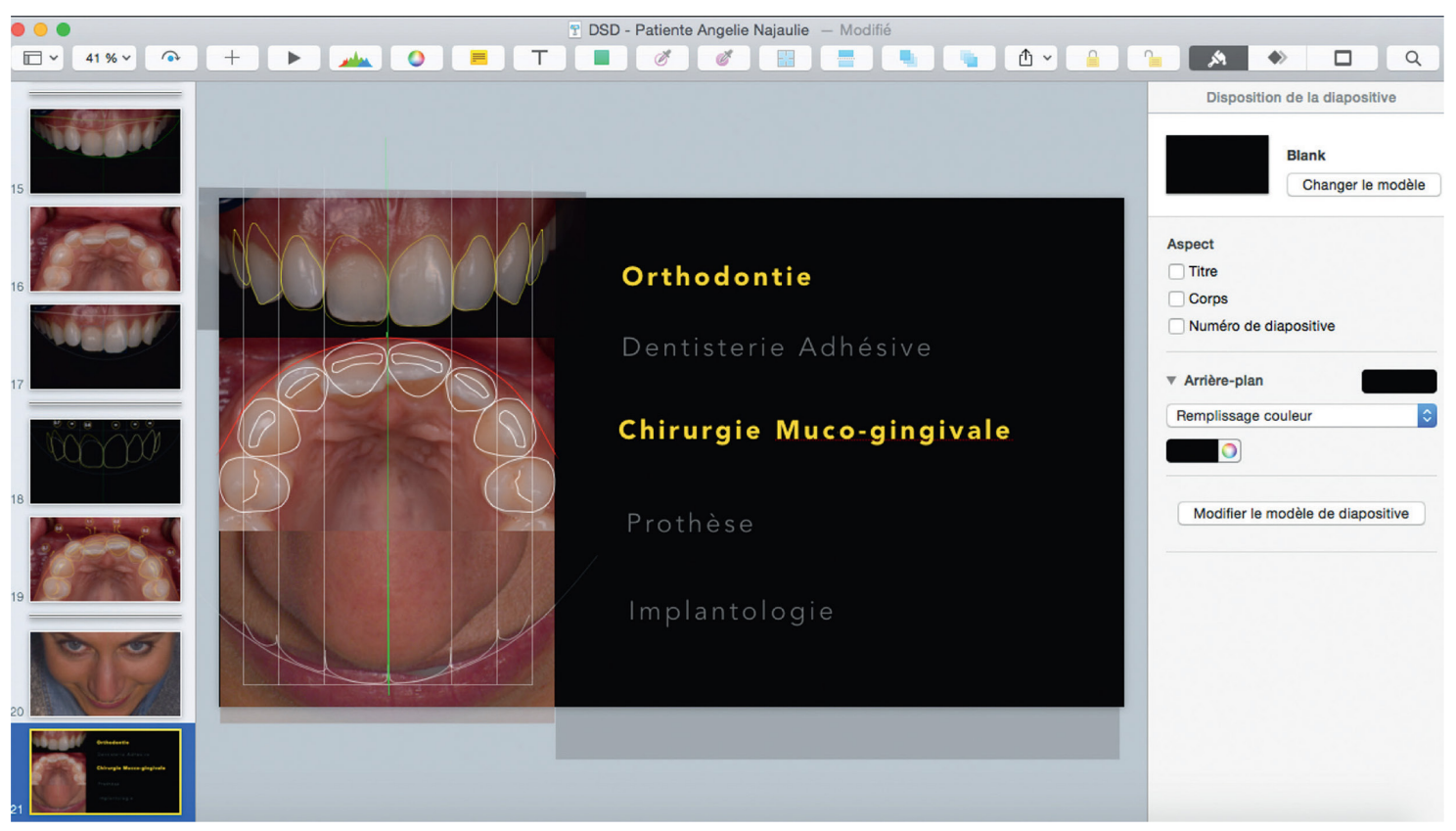

Figure 11

The last slide, the "smile frame" is a synthesis of the analysis and the end result of the project. It is made available to the entire treatment team and allows the different disciplines to prepare their treatment indications. 


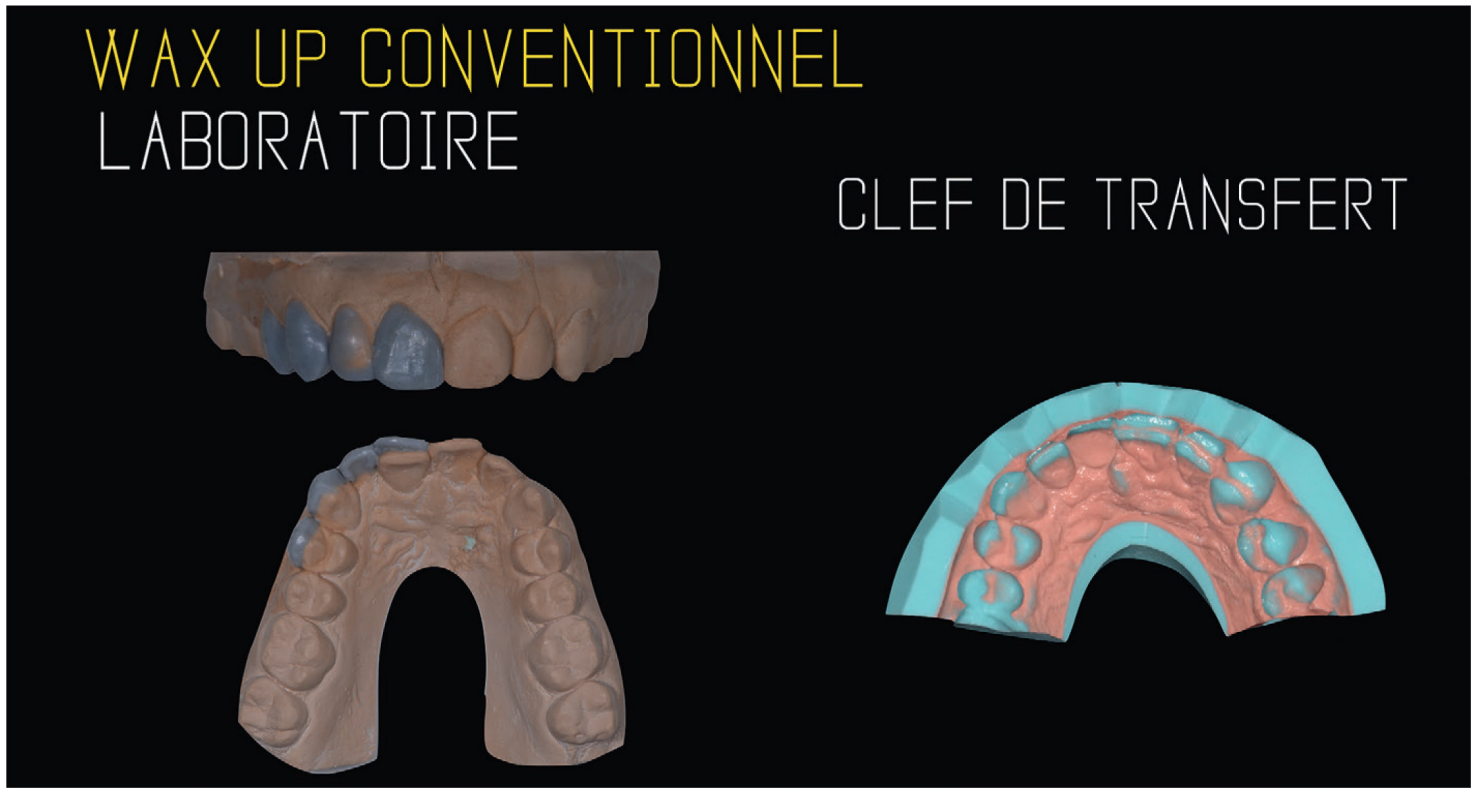

Figure 12

The established DSD is addressed to the laboratory prosthetist who can now guide the wax up by following the indications given by the project. A silicone cast is made to perform the mock-up or test drive before the start of the treatment (usually during the second appointment).

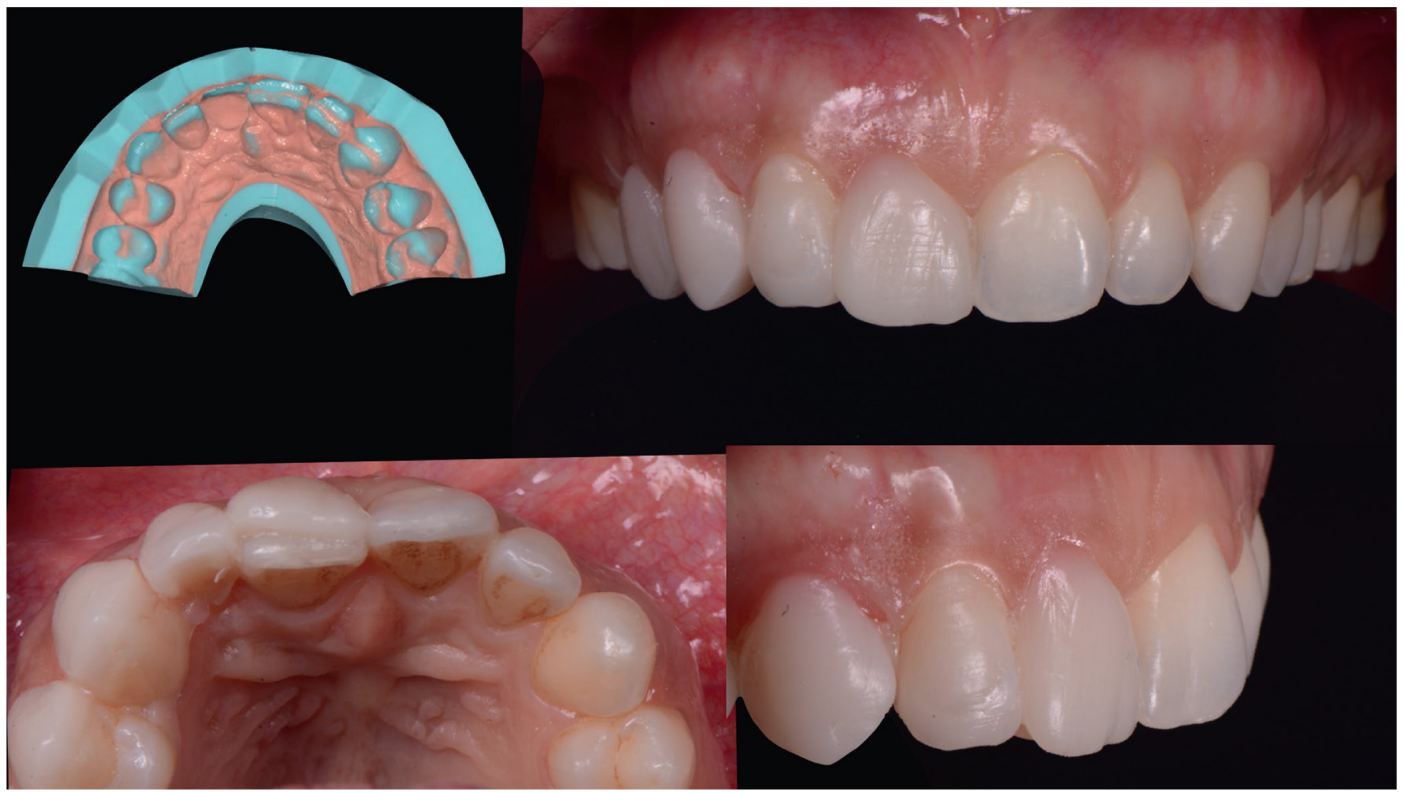

Figure 13

Once the wax up is done on the working mock-up with the prosthetic, it can be tested in the mouth in the form of mock-up (Bis-Acryl resin, Luxatemp) via a silicone cast. This key step in the protocol allows us to clinically validate our project in cooperation with the patient and to proceed with the treatment. 


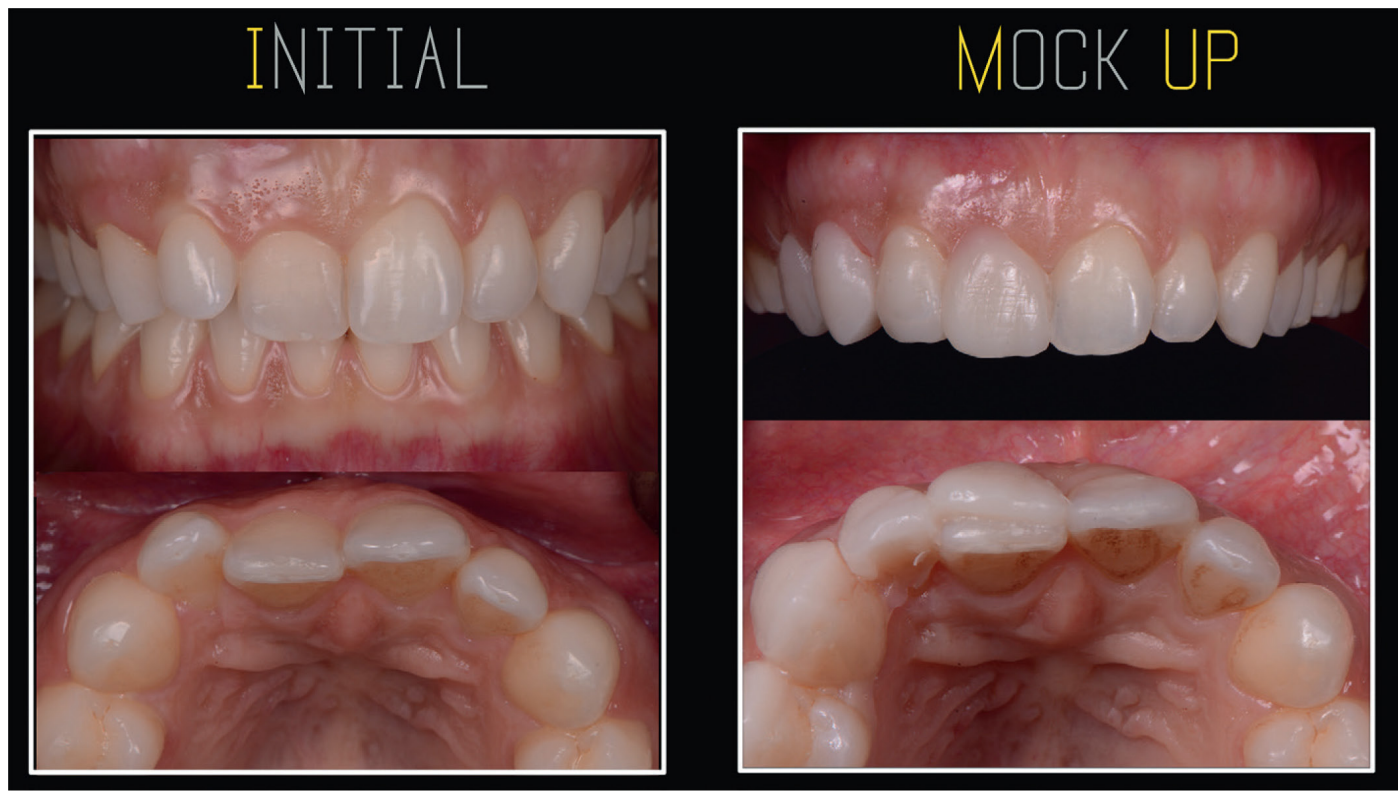

Figure 14

Initial vestibular and occlusal views (left). Vestibular and occlusal views after setting up the mock-up (right).
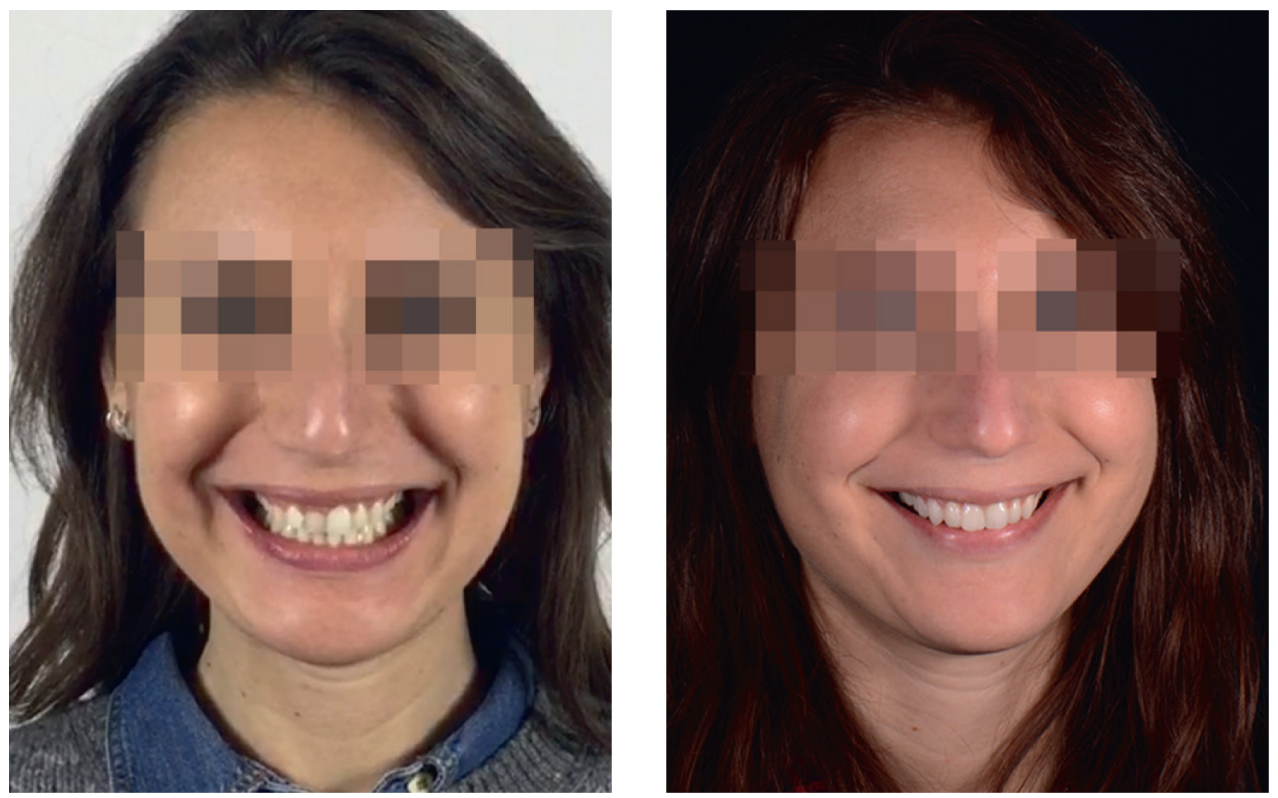

Figure 15

Face before treatment (left). Face with the mock-up in place (right). 
During the second appointment, a silicone cast (conventional technique; Fig. (12) allows the project to be tested clinically in the mouth and to evaluate any changes to be made or predict any compromises (Fig. (13) in the context of the face. The setting up of the mock- up is described as the most important step in the DSD protocol. It allows the patient to project themselves into their treatment and to appreciate the improvements to the smile (Figs. 14, 15). It is often at this point that the relationship of trust is established 9 .

\section{FROM 2D TO 3D: FROM THE DSD TO THE ORTHODONTIC ATTACHMENT}

Using an optical impression (Fig. (16) and 3D modeling software (Nemosoftware, DSD Connect, Cerec) the integration of the $2 \mathrm{D}$ project can now be directly changed into a $3 \mathrm{D}$ project in the form of three-dimensional virtual set up (Figs. 17, 18) ${ }^{10}$.

The 3D set up file can be exported to a 3D printer (Fig. 19) to create the resin mock-up and to complete the mock-up stage.

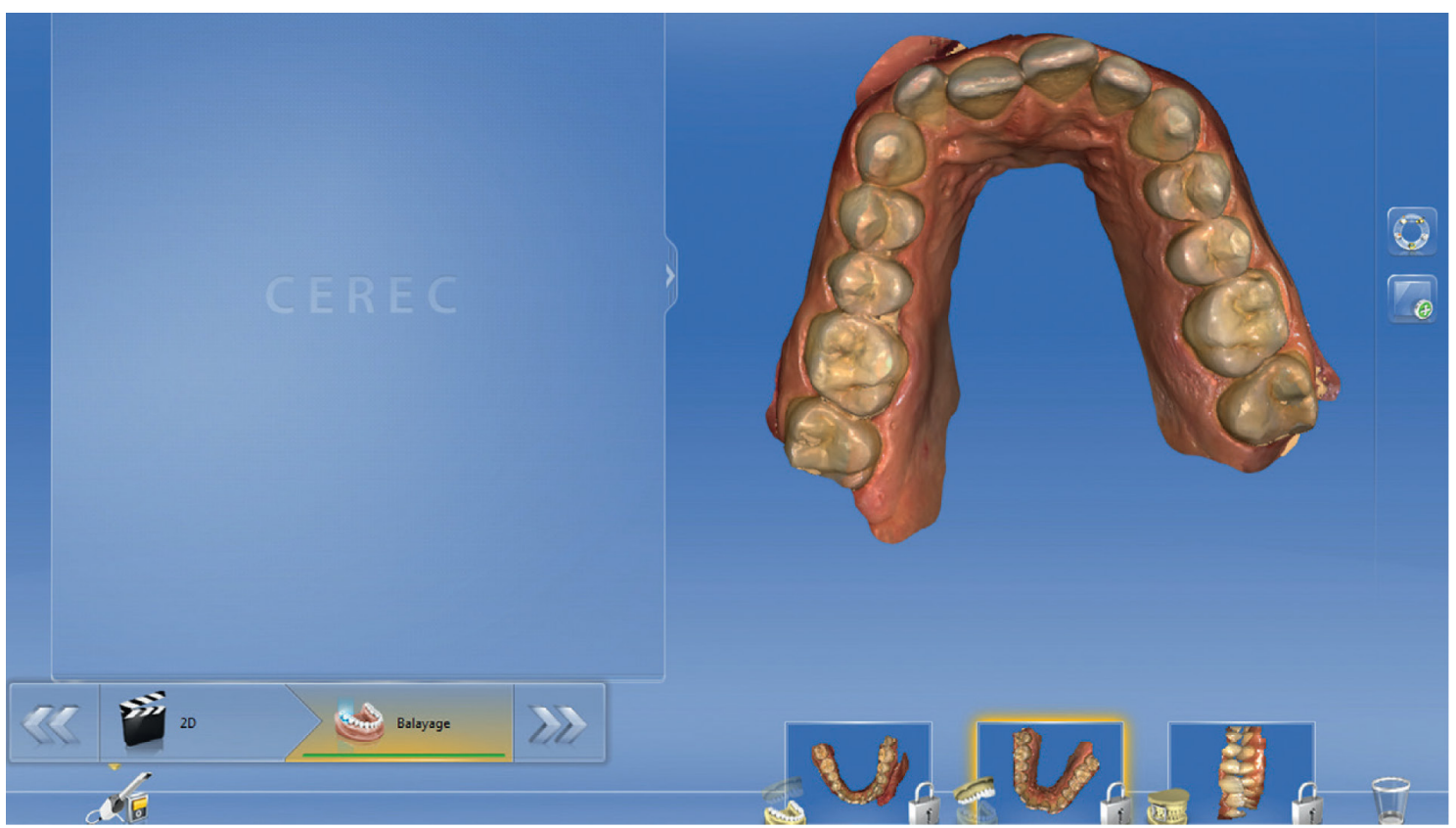

Figure 16

To optimize workflow connectivity, the 3D environment is used from the impression stage. An optical impression is made and then exported to software that will ensure the support of the previously established DSD. 


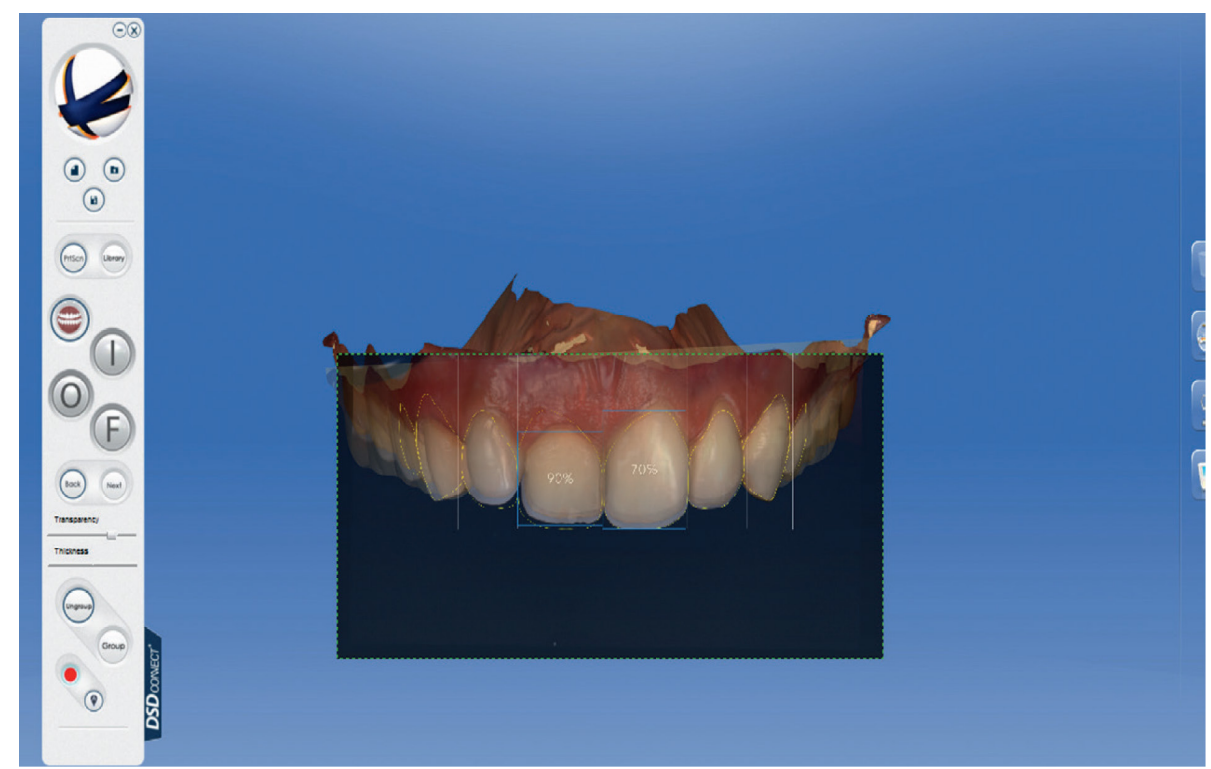

Figure 17

The transfer of the DSD 2D project to 3D consists of superposing the synthesis images of the $2 D$ project with the $3 D$ model from the optical impression in the three consolidated dimensions of DSD. The overlay of 2D files with 3D is carried out on using modeling software (prosthetic or orthodontic). When software does not provide for any facial integration (which is still often the case), DSD Connect software is used as a transfer. It works as a "mask screen" superimposing project (transparent) to guide the 3D mock-up.

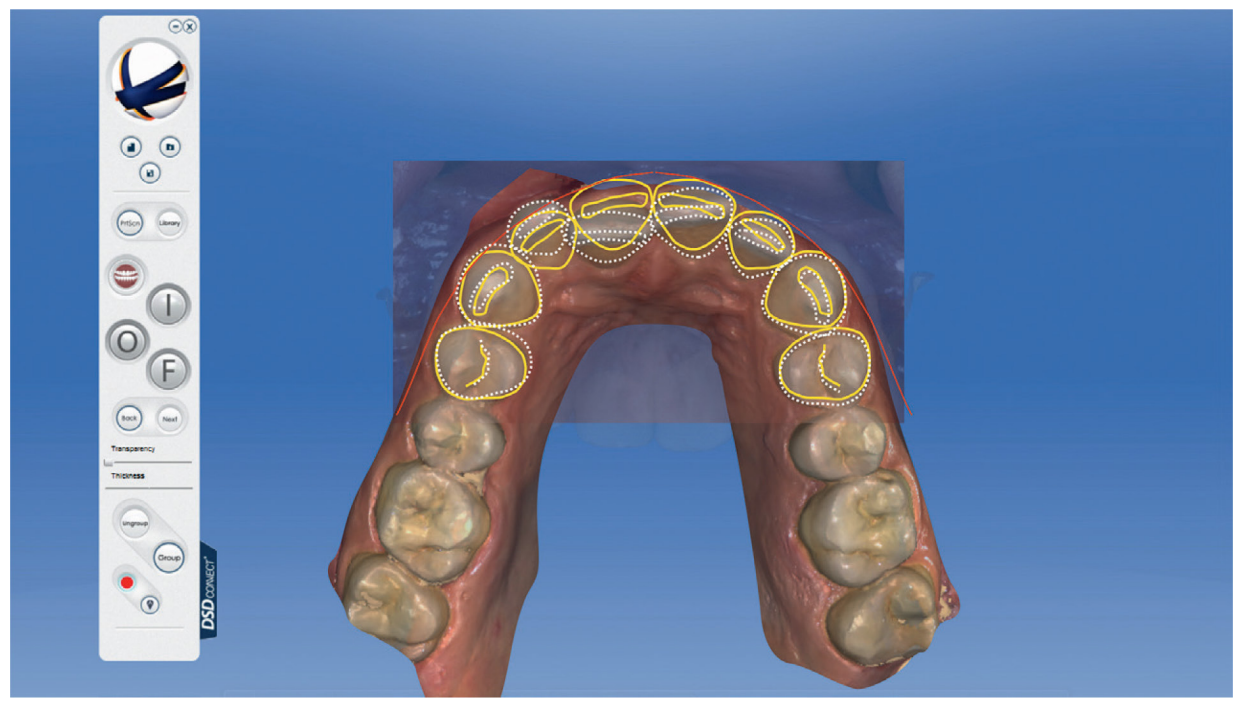

Figure 18

In occlusal view, the tooth-to-tooth virtual segmentation is performed and is shown here in white dotted lines and the project in yellow. DSD Connect software is used to make the orthodontic set up in accordance with the established DSD. The end product of this set up must be used for the manufacture of the orthodontist's device (Invisalign ${ }^{\circledR}$, Incognito ${ }^{\circledR}$. 


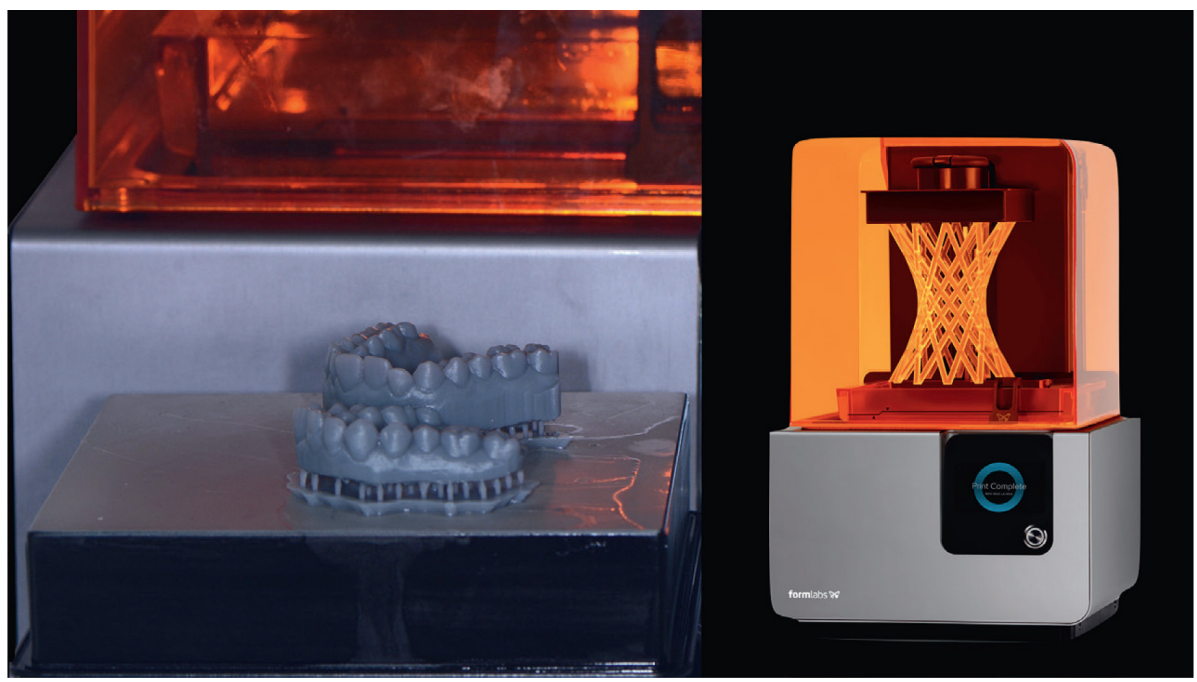

Figure 19

The diagnostic model and orthodontic set up are printed in gray resin using a 3D printer (Formlabs 2) in the dental practice.

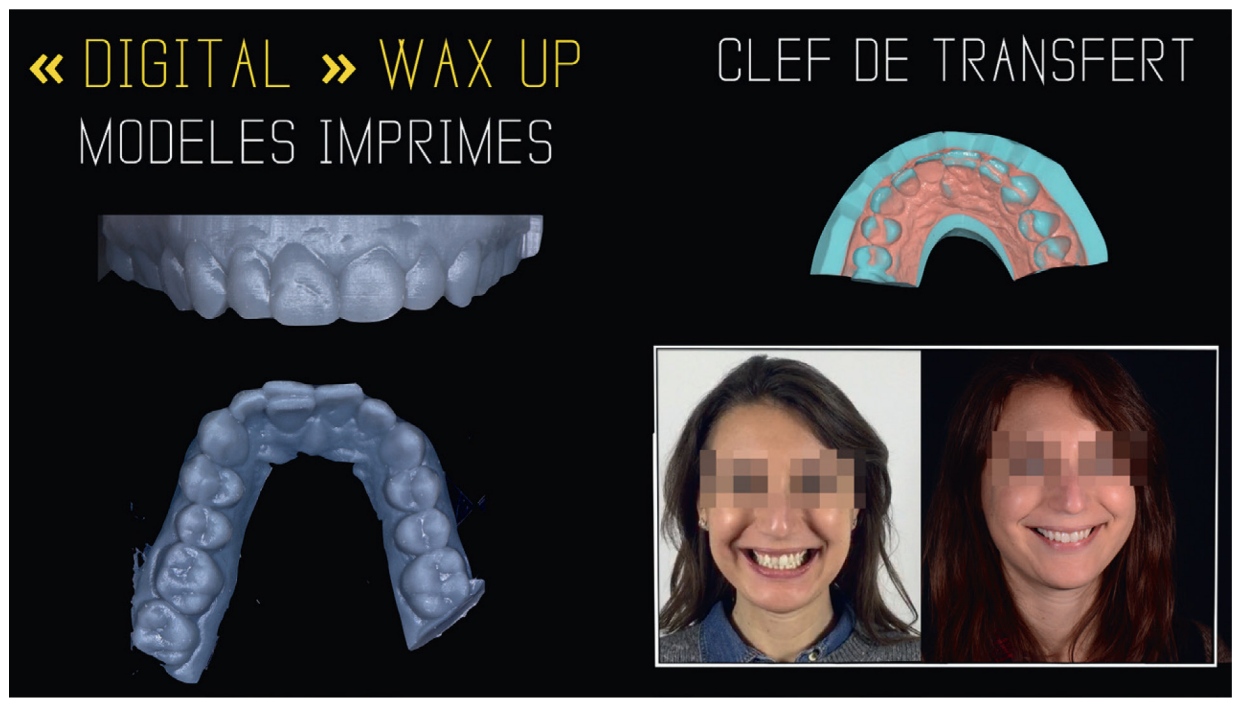

Figure 20

To carry out the mock-up digitally, the project is modeled and printed (the occlusal and palatal structures are preserved to facilitate the repositioning of the mock-up template). As with conventional techniques, a silicone cast is made (on the printed model) to transfer the project into the mouth. 


\section{FULL 3D ACQUISITION - FACIAL SCANNER}

The emergence of facial scanners in the dental industry and more specifically in orthodontics gives us a glimpse into the bright future of virtual diagnostics.

From facial scanners specifically designed for the dental industry (Zirkonzahn, Pritidenta, Planmeca; Fig. 21) or simple iPhone applications using a series of photos to perform 3D facial reconstruction (123Dcatch), the merging of clinical information (face, 3D model, cone beam) as well as smile design software can be done in 3D (modeling software) from the start of the analysis. This method would therefore come into effect from the transferring step from 2D to 3D ${ }^{12}$. Nevertheless, this strategy, although promising, is still in the experimental stage and is largely limited by the compatibility of orthodontic software.

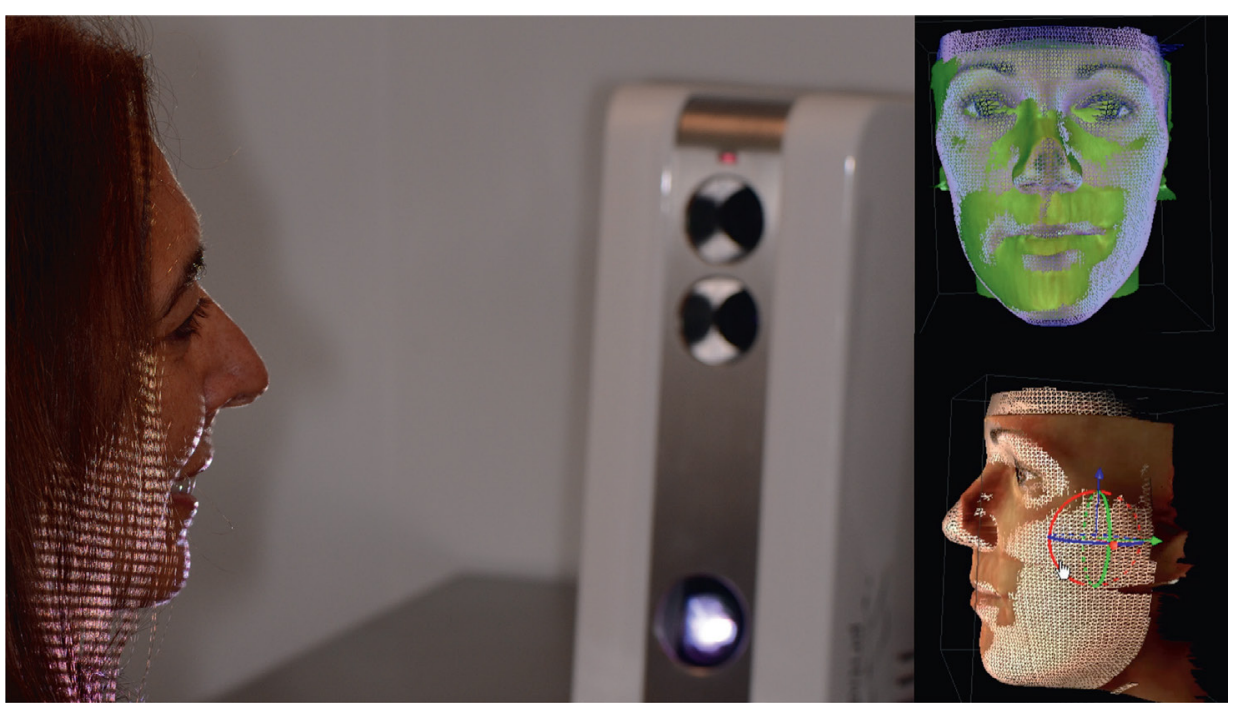

Figure 21

Facial scanners (Zirkonzahn, Pritidenta, Planmeca) allow you to record facial information directly in 3D. The smile design is therefore performed directly on a modeling software incorporating the work model and the three-dimensional facial representation of the face.

\section{DSD IN ORTHODONTICS}

Communication between the orthodontist and other members of the multidisciplinary team is one of the keys to therapeutic success in esthetic dentistry (Fig. 11).
DSD responds to three major objectives in the framework of orthodontic support:

- it allows the general practitioner to place an indication of an orthodontic 
Example 1: Periodontal development and adhesive dentistry (Figs. 22, 23, 24);
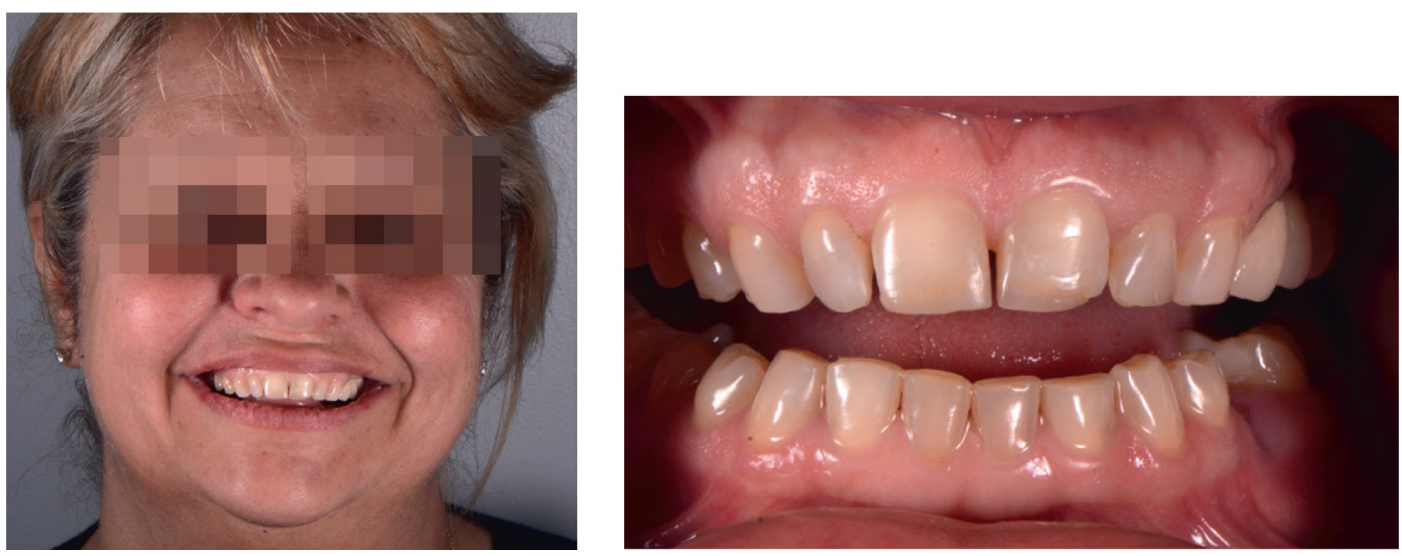

Figure 22

Example 1: initial situation. Patient consults for an esthetic rehabilitation request to correct the shape of disharmonious teeth and a marked gingival smile.

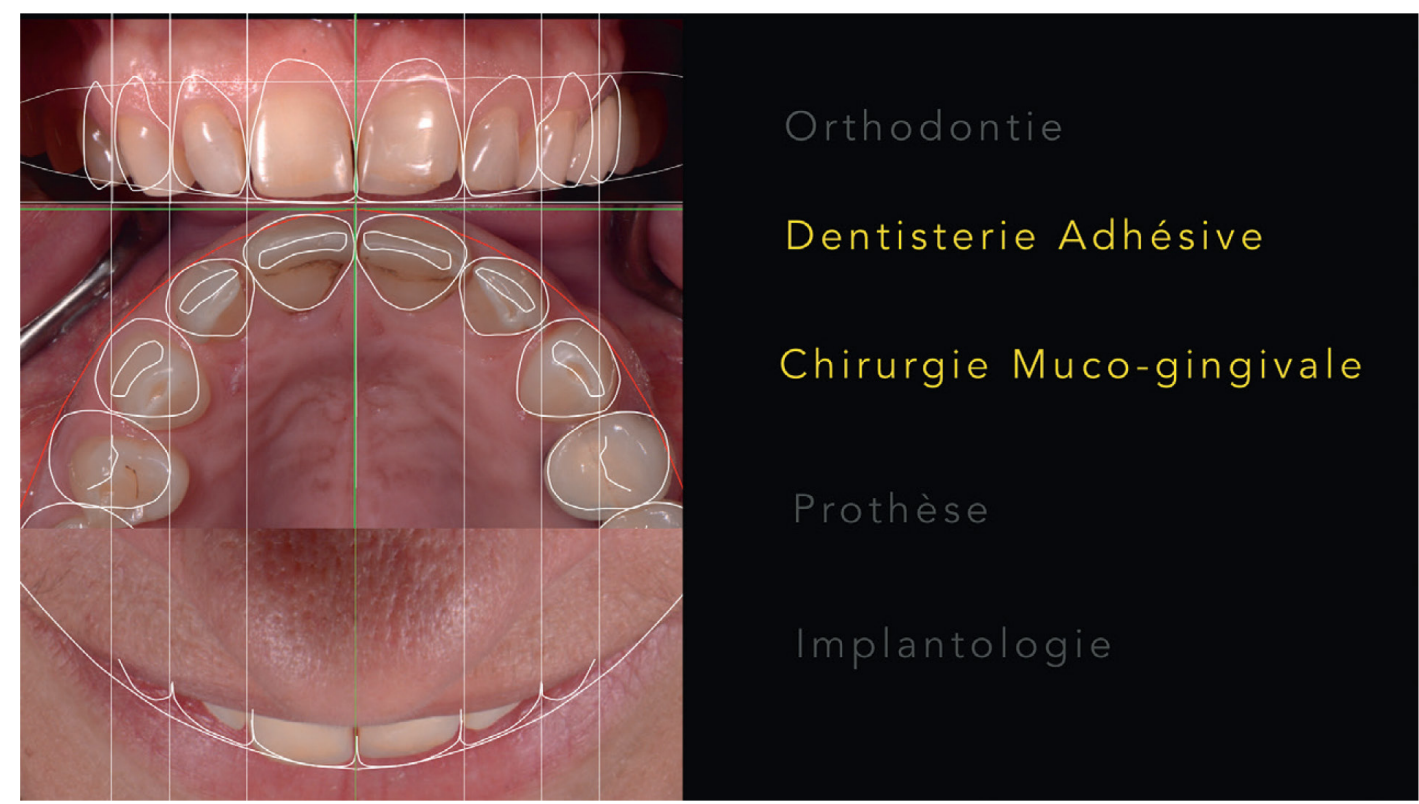

Figure 23

Example 1: Analysis shows a disharmony in the shape, proportion, and positioning of the necks. A minimally restorative esthetic treatment (facet type) combined with a mucogingival repositioning of the collars is recommended. 


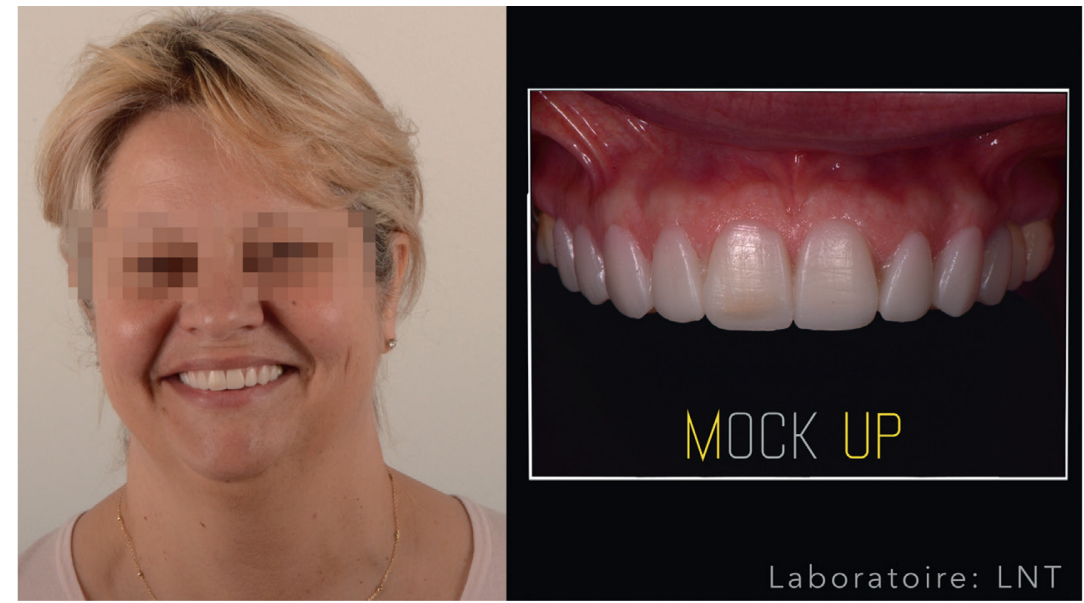

Figure 24

Example 1: Fitting after completing of the mock-up using the DSD project.

Example 2: Implant and prosthetic (Figs. 25, 26, 27);

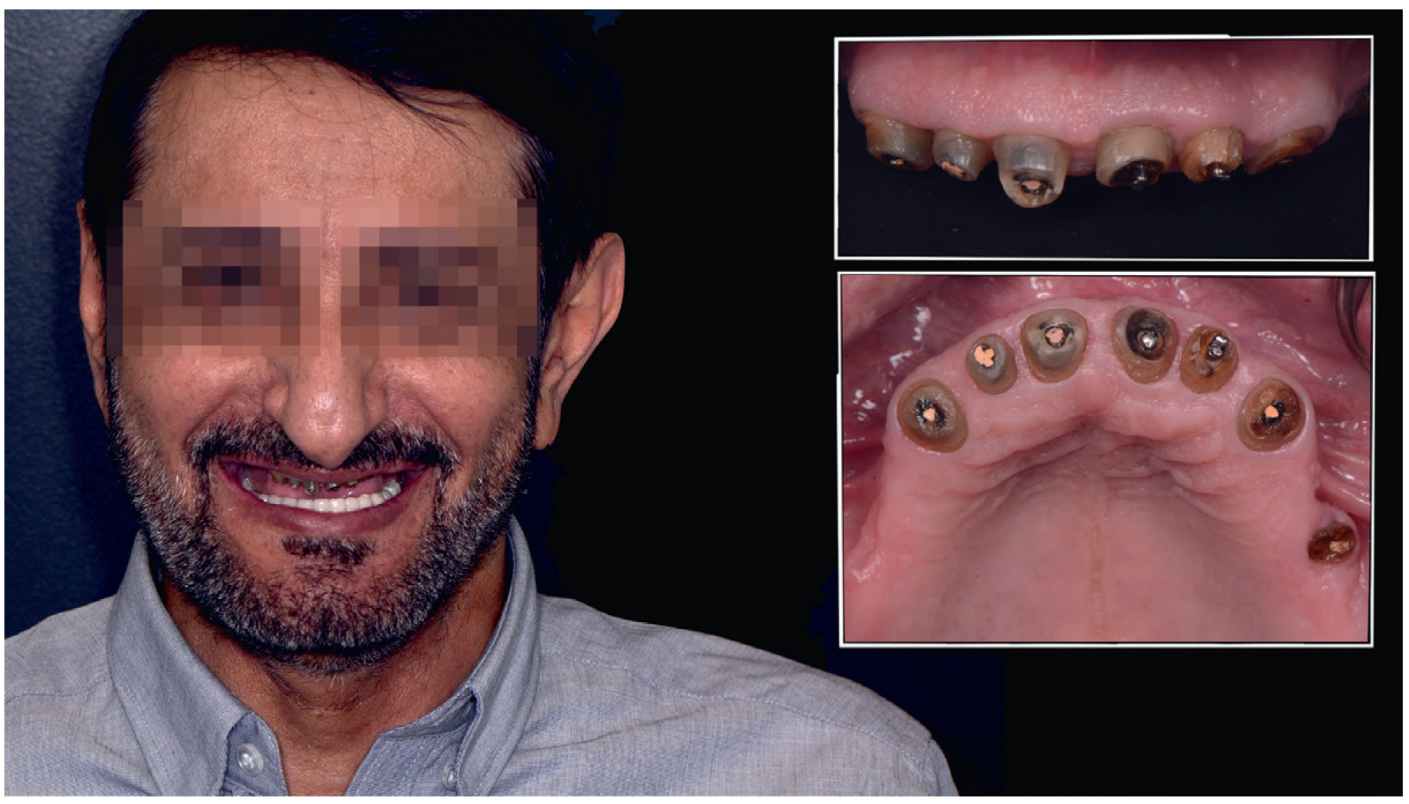

Figure 25

Example 2: initial situation. Patient consults for complete esthetic and functional rehabilitation. 


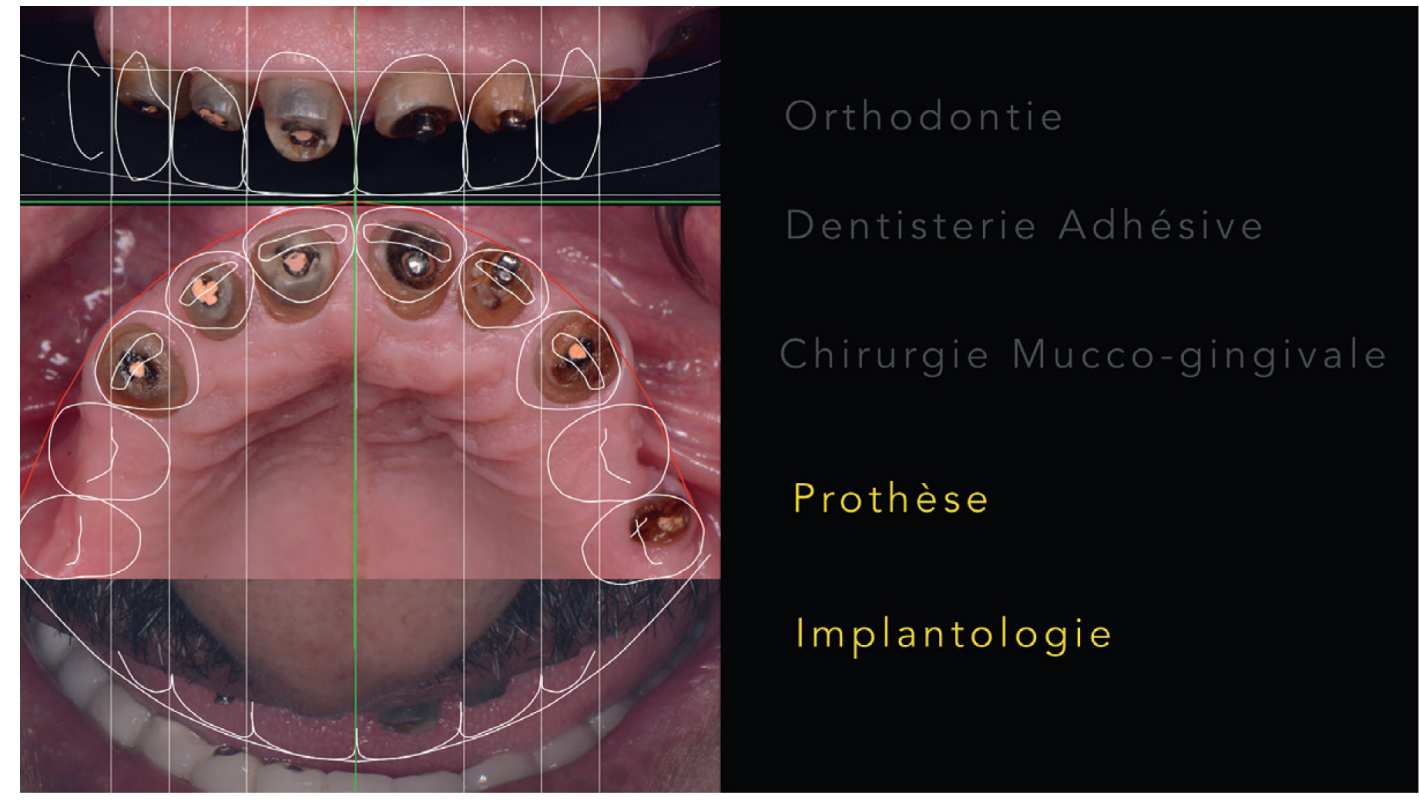

Figure 26

Example 2: The previously mentioned prosthetic project, Digital Smile Design, will allow implant planning to be performed in the most compatible manner possible with ideal esthetic rehabilitation.

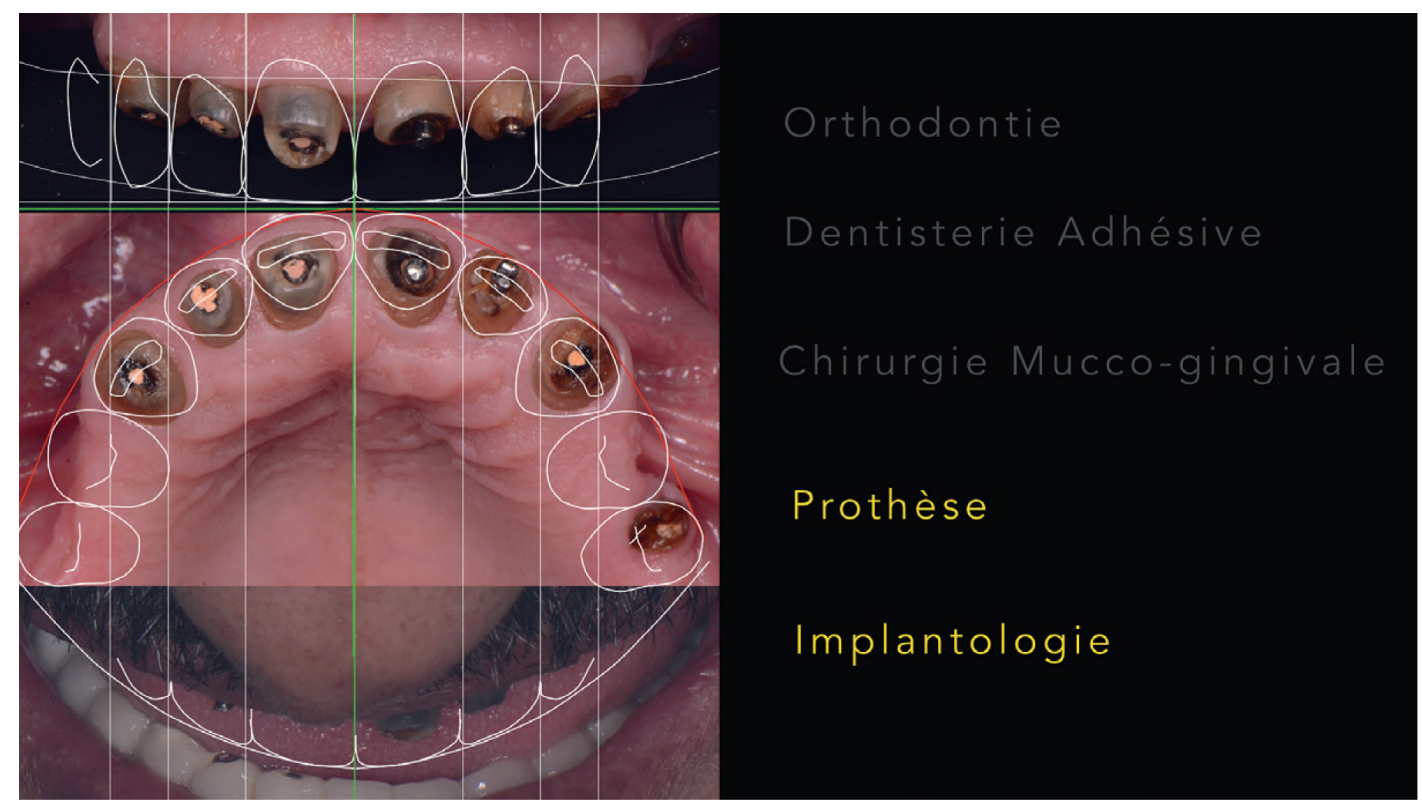

Figure 27

Example 2: Mock-up of the DSD project. 
Example 3: Orthodontics, implant, and prosthetics (Figs. 28, 29, 30).

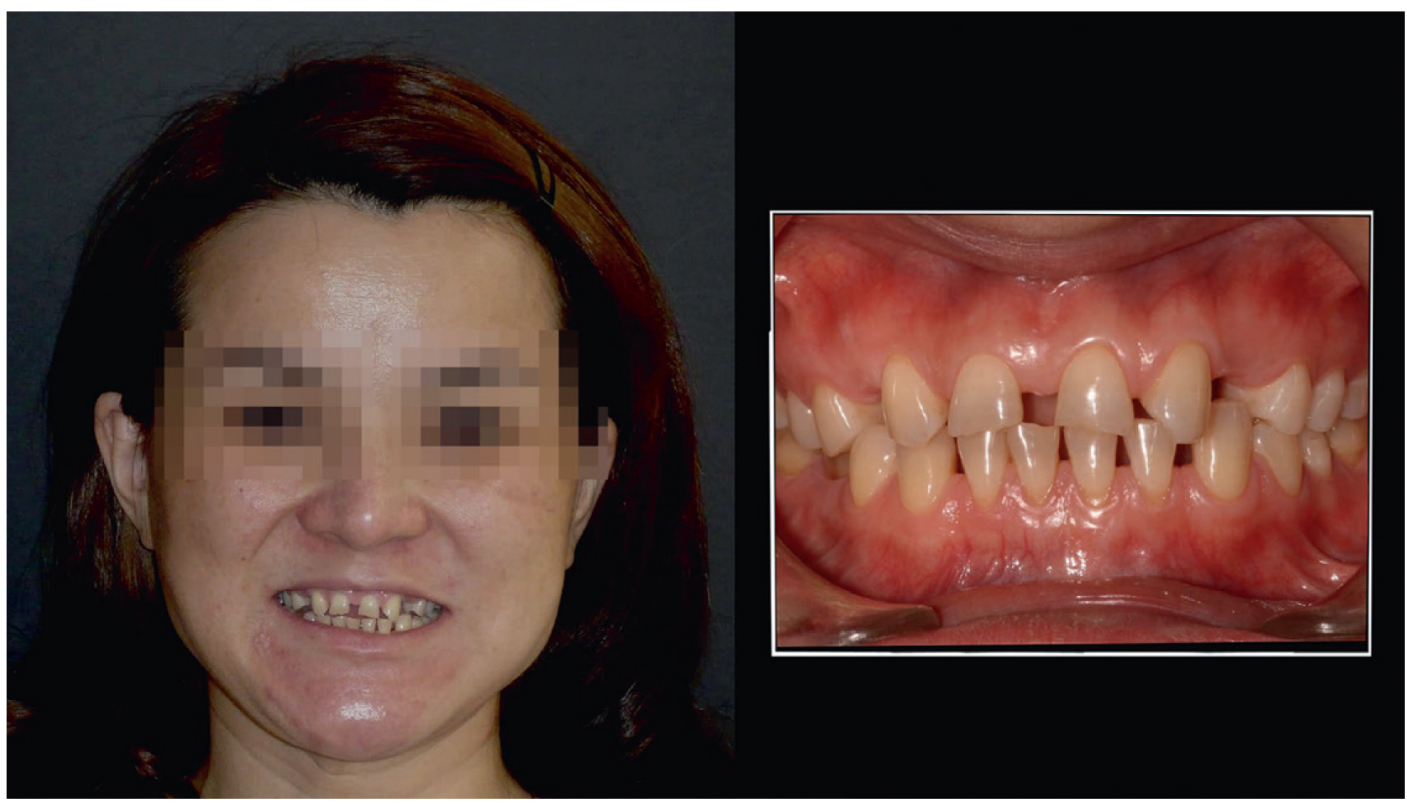

Figure 28

Example 3: initial situation. Patient consults for esthetic and functional rehabilitation.

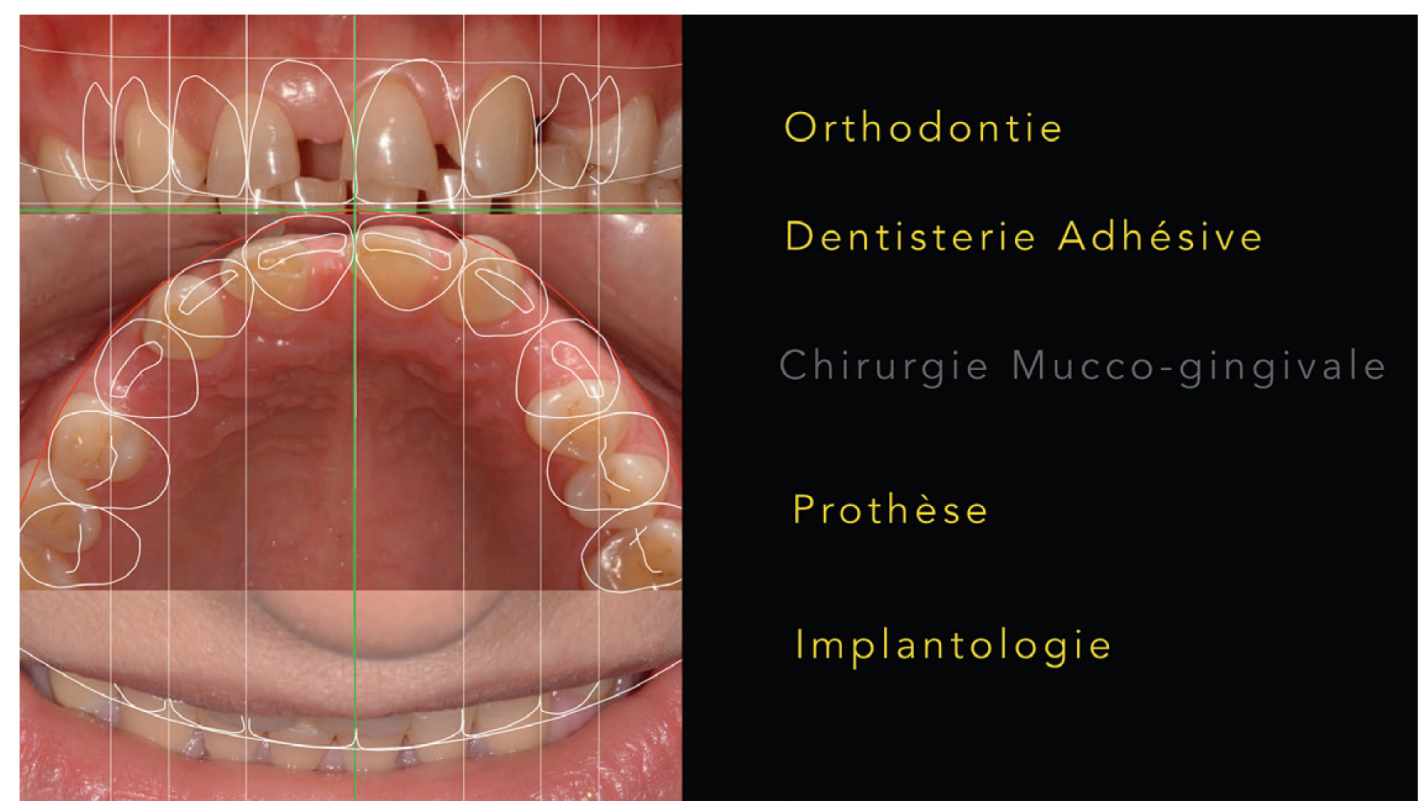

Figure 29

Example 3: the esthetic analysis reveals a disharmony linked to the absence of 12 and 22, malposition of the anterior teeth, incorrect position of the collars, a shift of the interincisal region (nonexhaustive list). The severity of the case indicates orthodontic support at first, followed by a phase of implant and prosthetic rehabilitation. 


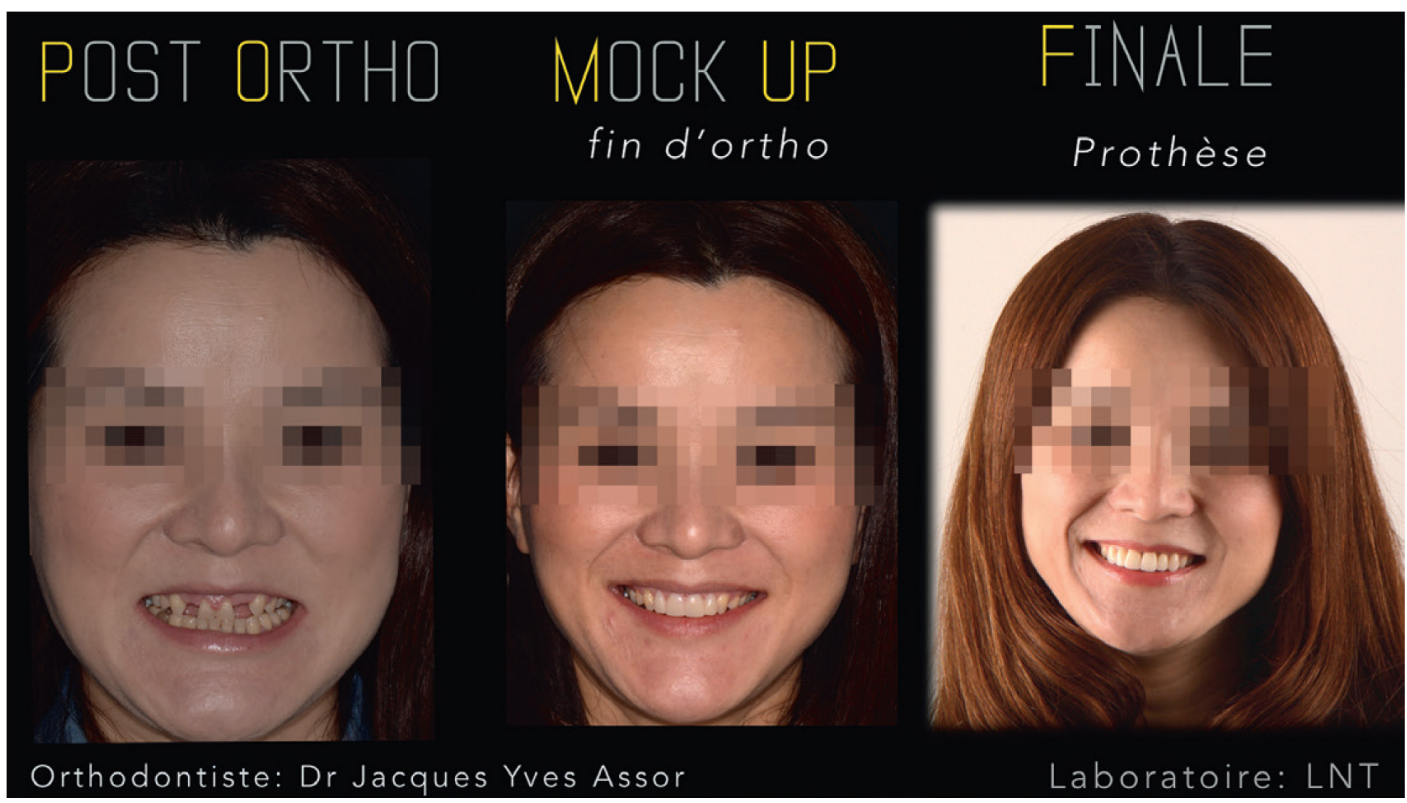

Figure 30

Orthodontic Development (Dr. Jacques Yves Assor) has restored the spaces and corrected the mispositions, guided by the ideal positioning indicated by DSD. A final orthodontic mock-up is done again to monitor the completed work and prepare for the implant-prosthetic phases. The right-hand photo was taken 6 months after the end of the implant and prosthetic treatment.

time (as a result of a contraindication of purely prosthetic care);

- it allows both parties to indicate the commonend-of-orthodontic-treatment objectives in the context of an associated prosthetic rehabilitation;

- it serves to validate the final positioning before the orthodontic appliance is removed.

It differs from the following methods:

\section{Orthodontic DSD without prosthetic support}

The purely orthodontic virtual project differs from the prosthetic project, in that it implies that only the position of the teeth will be modified, it also preserves the structural integrity of these teeth. This is why the summary plots represent the contour of the original teeth in their original and final positions (esthetic project; Fig. 8).

The overlap of the two situations illustrates the importance of the movements to be applied by the orthodontist from the three 2D views described previously. The case illustrated in this article belongs in this category. A revaluation at the end of the orthodontic treatment may cause the general practitioner to conduct minor complementary treatments (gingivectomy, composite, ceramic implants).

\section{Orthodontic DSD followed by prosthetic support}

Frequently, the management of esthetic rehabilitations in adult patients 
changes the position and shape of the teeth which takes up orthodontic and prosthetic (and/or implant) time. Under these circumstances, an orthodontic set up must be planned in line with the final prosthetic rehabilitation project in the context of minimally invasive dentistry.

Therefore, at the end of the orthodontic treatment, the volume of the displaced teeth should, as far as possible:

- be contained within the desired future volume of the prosthetic project;

- allow, in the case of adhesive restorations (facets), a prosthetic rehabilitation with a minimum amount of preparation at the expense of dental structures.

In this situation, two projects will be established:

- Project 1 (intermediate orthodontics): orthodontic exclusions (see above);

- Project 2 prefiguring how to achieve the restorations after orthodontic treatment.

In addition to the detailed case discussed in this article, three examples of multidisciplinary approaches guided by a virtual facial project are presented here to illustrate the therapeutic orientations taken before the start of treatment.

\section{CONCLUSION}

At a time when requests for esthetic rehabilitations and the demands of our patients are growing, it is up to practitioners to devise strategies to consolidate communication and avoid results that fall short of the expectations of patients or the practitioner themselves. Smile preview techniques are used to establish a predictable treatment plan based on facial analysis (not confined to inside the mouth). Constant and continuous developments in the field of digital orthodontics have led to interesting ideas for ensuring continuity between the design of the DSD project and the automated creation of orthodontic devices.

Conflict of Interest: The author states there is no conflict of interest.

\section{BIBLIOGRAPHY}

1. Coachman C, Calamita MA, Coachman FG, Coachman RG, Sesma N. Facially generated and cephalometric guided 3D digital design for complete mouth implant rehabilitation: A clinical report. J Prosthet Dent 2016 Nov 8.

2. Coachman C, Paravina RD. Digitally Enhanced Esthetic Dentistry - From Treatment Planning to Quality Control. J Esthet Restor Dent 2016;28 Suppl 1:S3-4.

3. Coachman C, Van Dooren E, G ürel G, Landsberg CJ, Calamita MA, Bichacho N. Smile design: From digital treatment planning to clinical reality. Interdisciplinary Treatment Planning. Vol 2: Comprehensive Case Studies. Chicago : Quintessence, 2012:119-174. 
4. Crescenzo H, Crescenzo D. Le Projet virtuel esthétique - Un nouvel outil pour les traitements esthétiques - Stratégie prothétique mai- juin 2015;(15)3.

5. Finelle G, Lehmann N, Coachman C. Technique de prévisualisation du sourire dans la réhabilitation implantaire du secteur antérieur. Rev Odont Stomat 2015;44:162-174.

6. Fradeani M. Esthetic Analysis: A Systematic Approach to Prosthetic Treatment. Esthetic Rehabilitation in Fixed Prosthodontics. Vol 1. Chicago : Ed. Quintessence, 2004. Coachman C, Calamita MA Digital Smile Design: A Tool for Treatment Planning and Communication in Esthetic Dentistry. Chicago : Quintessence, 2012.

7. Goldstein RE. Esthetics in dentistry: principles, com munication, treatment methods. Ed. B.C. Decker, 1998.

8. Gürel G, Bichacho N. Permanent diagnostic pro visional restorations for predictable results when redesigning smiles. Pract Proced Aesthet Dent 2006;18(5):281-6.

9. Kurbad A, Kurbad S. Cerec Smile Design--a software tool for the enhancement of restorations in the esthetic zone. Int J Comput Dent 2013;16(3):255-69.

10. Levrini L, Tieghi G, Bini V. Invisalign ClinCheck and the Aesthetic Digital Smile Design Protocol. J Clin Orthod 2015;49(8):518-24.

11. $M$ agne $P$, Belser $U$. Restaurations adh ésives en céra mique sur dents antérieures : Approche biomimétique. Paris: Quintessence International, 2003.

12. Zimmermann M, Mehl A. Virtual smile design systems: a current review. nt J Comput Dent 2015;18(4):303-17. 